\title{
Género, vulnerabilidad y precariedad de los jóvenes de la Ciudad de México en la reproducción de la desigualdad social
}

\section{Gender, vulnerability and precariousness of young people in Mexico City in the reproduction of social inequality}

doi: http://dx.doi.org/10.32870/

Miguel Ángel Vite Pérez

espiral.v25i71.5591

\begin{abstract}
Resumen
Este artículo analiza, desde un punto de vista teórico, la elaboración de una explicación sobre la vulnerabilidad social juvenil mexicana según algunas evidencias estadísticas. Se muestra la debilidad de las instituciones, que han sido favorables a la reproducción de la desigualdad de género, vinculada, al mismo tiempo, al contexto de pobreza y desigualdad social. Se concluye que en la Ciudad de México la precariedad juvenil se reproduce de diversas formas a pesar de la institucionalización de los diferentes programas de asistencia social.
\end{abstract}

Palabras clave: vulnerabilidad social, precariedad juvenil, protecciones sociales, asistencia social, género.

\begin{abstract}
This paper analyzes, from a theoretical point of view, the development of an explanation of Mexican youth social vulnerability through some statistical evidence, which showed the weakness of institutions, which have been favorable to the reproduction of gender inequality that is linked at the same time to the context of poverty and social inequality. It is concluded that in Mexico City precarious youth plays in various ways despite the institutionalization of the different social assistance programs.
\end{abstract}

Keywords: Social vulnerability, youth insecurity, social benefits, social welfare, gender.

\footnotetext{
- Profesor-Investigador del Instituto Politécnico Nacional (IPN), México. ORCID: http://orcid. org/0000-0002-8799-44449.

miguelviteperez@yahoo.com.mx

Fecha de recepción: 20 de mayo de 2016. Fecha de aceptación: 27 de octubre de 2017
} 


\section{Introducción}

El propósito general del presente artículo es elaborar una interpretación sobre la relación entre género y vulnerabilidad basada en algunos puntos de vista teóricos, para explicar la precariedad juvenil, fomentada por el crecimiento de las desigualdades sociales, consideradas a su vez como la causa de la descomposición del vínculo social, es decir, de la solidaridad, sobre todo porque las brechas entre ingresos han aumentado y también la concentración de los patrimonios (Rosanvallon, 2012, pp. 18-19).

El concepto de género resulta de utilidad para el análisis de la precariedad juvenil porque evidencia las desigualdades entre hombres y mujeres que han sido reproducidas por las instituciones sociales y se han convertido en el eje de una identidad, basada en el sexo (Giacomello, 2013, p. 40).

Por tal motivo, la idea de género, que se deriva de la existencia de normas heterosexuales, configura un régimen con reglas que definen los atributos de hombres y mujeres en una sociedad a través de la coerción y la violencia contra aquellos que no las acatan, por ejemplo, porque sus comportamientos expresan orientaciones e identidades como la homosexualidad, el travestismo o el lesbianismo (Butler, 2014 pp. 25-26).

Sin embargo, los sujetos definidos por las estructuras heterosexuales (como oposición binaria masculino-femenino) son significados culturales que aceptan el cuerpo sexuado como prediscurso, es decir, "[la] producción del sexo como lo prediscursivo debe de entenderse como el resultado del aparato de construcción cultural nombrado por el género" (Butler, 2014, p. 56).

En este sentido, y de acuerdo con Butler (2014, p. 49), el género se produce y conserva en una sociedad porque se liga con diferencias de diverso tipo como de clase, étnicas, sexuales y de identidad, esto en contextos históricos diferentes. 
En consecuencia, el concepto de género no sólo permite estudiar las diversas formas de subordinación basadas en la idea de la superioridad de lo masculino, sino también algunas de las consecuencias desprendidas de esa relación asimétrica, como lo es la desigualdad social, que ha creado situaciones de pobreza y exclusión y de violencia que afectan más a la parte vulnerable de la relación subordinada, a saber, a las mujeres, por lo que dicha relación configura un sistema de dominación (Vendrell, 2013, p. 14).

Una manifestación de la desigualdad social contemporánea es la precariedad, que no sólo se vincula con la vulnerabilidad creada por la existencia de un empleo o trabajo con bajos ingresos y con escasas o nulas protecciones sociales -garantizadas como derechos sociales-, sino también con la existencia de individuos sin empleo, que viven de la caridad pública y privada, y que no cuentan con un reconocimiento social porque se considera que no son funcionales a la dinámica socioeconómica, esto último, sobre todo, porque han roto todo lazo o vínculo con las instituciones sociales. Esto diferencia a esta clase de individuos del llamado pobre, el cual ha sido definido socialmente para alcanzar un reconocimiento que le permite acceder a los programas estatales de ayuda social (Le Blanc, 2007, p. 79).

Entonces, las construcciones sociales y simbólicas que conlleva el concepto de género se han articulado a un nuevo contexto, caracterizado por la desigualdad social. En otras palabras, han dado lugar a una desigualdad de género, que desde un punto de vista general impide que funcione una estructura de posibilidades transformadas en oportunidades y reconocimientos, y relacionadas con los principios de igualdad, justicia, autonomía, respeto y derechos (Zaremberg, 2013, p. 15).

La idea de desigualdad de género puede interpretarse con la ayuda de los conceptos vulnerabilidad y precariedad, que emergieron en un nuevo contexto, caracterizado por la 
desvinculación paulatina de los derechos sociales del trabajo asalariado (Castel, 2010, pp. 15-55).

Es necesario destacar que el papel de las instituciones sociales en dicho contexto era el de evitar la ampliación de la desigualdad social a través de mecanismos de integración social, que dotaban a los individuos de atributos sociales que les permitían gozar de condiciones materiales de posibilidad para llegar a ser individuos sociales, independientes y con derechos (Valero Matas, 2009, pp. 69-70; Castel y Haroche, 2003, pp. 35-37).

Por otro lado, desde una justificación metodológica, los conceptos teóricos posibilitan la elaboración de interpretaciones sobre un hecho convertido en objeto de la reflexión al considerar nuevos elementos que han transformado la realidad social, como es el caso del tema de género y juventud (Aguilar Gil, 2010, pp. 135-142).

En el contexto de los jóvenes mexicanos, su vulnerabilidad y precariedad tienen también como causa la baja capacidad de integración de las instituciones mexicanas, que se ha intentado solucionar a través de los programas de asistencia social focalizada, cuya selectividad implica excluir de sus beneficios a otros cuyas necesidades no han sido reconocidas como parte de la extrema pobreza. Esto incluye a una franja importante de jóvenes que luego buscan alternativas de excepción para satisfacer sus necesidades por medio de la economía informal o a través de actividades ilícitas (Paugam, 2007; Bauman, 2008, pp. 9-17).

En México, la condición de vulnerabilidad juvenil no significa vigencia de derechos sociales, sino asistencialismo y relaciones sociales frágiles, las cuales no han alcanzado para construir sentido de pertenencia o adscripción con la sociedad en los jóvenes. Ante esto, una de las alternativas para algunos de estos ha sido la construcción de lazos de pertenencia a grupos juveniles con identidades particulares (Valenzuela, 2009, p. 37).

\section{6}


Metodológicamente, a través de sus percepciones y opiniones, se puede entender cómo los jóvenes mexicanos viven su vulnerabilidad y precariedad en un contexto de desigualdad social en que esta ha sido reproducida desde las instituciones orientadas por la directriz heterosexual. Por ello, para visualizar lo anterior, se analizan aquí los datos recogidos en la Consulta de tendencias juveniles 2013, Ciudad de México (Instituto de la Juventud del Distrito Federal, 2013). En ella, por ejemplo, la desigualdad de género es observada a través de la construcción de una categoría, juventud, que hace imposible distinguir las opiniones de hombres y mujeres, acorde con una idea de igualdad que neutraliza las diferencias. En otras palabras, el hombre y la mujer son reconocidos como semejantes, partiendo del supuesto que son individuos iguales en derechos (Rosanvallon, 2012, pp. 324-325).

Por otro lado, la desconfianza que expresan los jóvenes que habitan la Ciudad de México es resultado de un proceso de socialización débil, donde los estudios escolares, por ejemplo, son vistos como un medio sólo para mejorar sus ingresos y no para fortalecer los compromisos sociales. De acuerdo con nuestra perspectiva, lo anterior también es resultado de una crisis de la creencia en la igualdad, derivada de la ocupación de posiciones en el lugar de trabajo, ahora definida por la igualdad de oportunidades y antes por los derechos que adquiría el individuo cuando ocupaba un puesto laboral (igualdad de posiciones).

De acuerdo con Dubet (2011, pp. 23-54), la igualdad de posiciones se vinculaba con la existencia del Estado social y sus políticas de redistribución, que otorgaban derechos a las posiciones derivadas de las relaciones asalariadas. Ahora, la igualdad de oportunidades se define independientemente de quién ocupe las posiciones, porque se parte del supuesto de que todas las clases sociales tienen la misma oportunidad de 
ocupar un puesto laboral, con una diferencia que proviene solamente del mérito logrado por la educación.

La idea de igualdad de oportunidades también ha contribuido a la reproducción de la desigualdad de género, pues se mantiene un acceso desigual de hombres y mujeres a las ventajas sociales, justificado ahora por la existencia de méritos (Ariza y De Oliveira, 2001, pp. 1-2). Por eso, la desigualdad de género ha encontrado su legitimación no solamente en el mantenimiento de la desigualdad sexual del trabajo, sino en la creencia en la igualdad de oportunidades, lo cual ha reforzado la idea de que las desigualdades son justas cuando dependen de los conocimientos y habilidades que poseen los individuos, independientemente de su sexo (aquí entra la consideración de la igualdad abstracta). Por otro lado, los jóvenes se transforman en actores sociales no sólo por los procesos de socialización tradicional (a través de la familia y la escuela), sino por situaciones diversas vinculadas, por ejemplo, con el narcotráfico, la migración, el género y la economía informal (Reguillo, 2012, p. 13).

El presente artículo se dividió en cuatro partes. En la primera, se revisa el concepto de vulnerabilidad social para estudiar la importancia de la integración social como un medio para evitar la expansión de la desigualdad social. En la segunda parte, se estudia el concepto de precariedad social según su significado en la configuración de una juventud vulnerable, que ha favorecido la reproducción de la desigualdad de género. En la tercera parte, y con ayuda de los conceptos de vulnerabilidad y precariedad social elaborados, se interpretan algunas opiniones de jóvenes que habitan la Ciudad de México para mostrar que la idea de igualdad de género es una manera de conservar el orden social heterosexual, apoyado en la idea de la igualdad de oportunidades. Finalmente, en la cuarta parte se presentan las conclusiones articuladas a través de la siguiente consideración: la desigualdad social juvenil se vincula con 
la de género, lo que también ha favorecido la aparición de situaciones de disolución social.

\section{La vulnerabilidad social como fragilidad de la solidaridad}

En las sociedades desarrolladas, el paradigma de la sociedad del trabajo tenía como principal fundamento los derechos sociales porque la condición de asalariado, es decir, el posicionamiento del individuo en la organización del trabajo productivo, estaba protegida por un sistema de bienestar estatal, considerado a su vez como la única forma de realización de los derechos sociales (Ochando Claramunt, 2002, pp. 27-30).

El trabajo asalariado adquirió importancia por la universalización de los derechos sociales hacia todo tipo de trabajo asalariado; en consecuencia, la desigualdad social no se derivaba sólo del monto de los salarios, sino de la falta de protecciones sociales (Castel, 2004a). En este sentido, lo social fue relacionado con la existencia de un sistema de bienestar estatal que hacía compatibles las desigualdades económicas capitalistas con el principio de igualdad democrática, en otras palabras, la obligación del Estado para reorganizar a la sociedad de acuerdo con principios igualitarios expresados como derechos creadores de solidaridad (Donzelot, 2007, pp. 50-53).

El asunto de la igualdad social fue una tarea esencial del llamado Estado social llevada a cabo por medio de mecanismos o dispositivos que generaban sentido de pertenencia de parte de los individuos a determinados colectivos, creando solidaridad, lo que reforzaba la integración social (Castel, 2010, pp. 23-30). Esto fue destruido por el nuevo régimen del capitalismo neoliberal, que buscó la construcción de una competencia individual sin responsabilidad estatal, aunque ligada a las posibilidades construidas por los méritos perso- 
nalizados (Steger y Roy, 2011, pp. 44-86; Chul Han, 2013, pp. 11-23).

En el contexto descrito, apareció una sociedad de individuos en la que el proceso de individualización se caracterizó por respuestas personales a los desafíos que tenían más un origen social. Derivado de esto, se consideró que había una crisis de las organizaciones y de las instituciones, las cuales fueron vistas de manera negativa al considerarlas como limitaciones o constricciones para el desarrollo de las capacidades del individuo. Lo anterior, de acuerdo con Castel (2010, pp. 26-27), creó dos tipos de individuos: el individuo definido por el éxito y el individuo por defecto, este último sometido a la precariedad permanente y a la invalidez social.

En el lenguaje de Castel (2004b, pp. 13-24), el individuo alcanzaría su autonomía cuando existieran las condiciones o soportes de identidad, por ejemplo, la propiedad, las protecciones sociales y los derechos al trabajo. Entonces, la sociedad de individuos significaría despojo de atributos sociales para algunos individuos que vivirían su existencia de manera precaria y degradada y sin ninguna protección estatal (Bauman, 2013, pp. 21-50).

El despojo de atributos sociales o identidad social también podría significar criminalizar a los individuos que han encontrado una funcionalidad en el crimen o en las actividades económicas de sobrevivencia, reproductoras de la pobreza y la miseria (Wacquant, 2000, pp. 21-24). En consecuencia, tanto Wacquant (2000) como Agamben (2007) hasta cierto punto coinciden en que el proceso de pérdida de atributos sociales crea individuos que pueden ser considerados como enemigos del orden estatal porque viven en una situación de excepción, donde no hay soportes o condiciones objetivas de posibilidad. Así, la situación de excepción individual significaría ausencia de atributos sociales manifestada en la escena pública como eventos molestos (la 
violencia, la enfermedad, la muerte, el sufrimiento), que se suponía antes se escondían en los dominios privados de la vida social, como la familia (Garland, 2007, p. 171).

La integración social vía el trabajo asalariado también mostró que la justicia social significaba distribución justa de posiciones o lugares a través de la compactación de la jerarquía de ingresos, que de acuerdo con Dubet (2013, pp. 166-167) concretaba la aspiración de mejora de condiciones de vida y de trabajo.

La solidaridad producía sociedad a través de la existencia de organizaciones, que moldeaban las subjetividades de sus miembros mediante su integración. Esto ha sido gradualmente abandonado para sustituirlo por la cohesión, bajo la cual los individuos están ligados sólo por acciones recíprocas, generadoras de identidades particulares, y donde la socialización efímera une a los individuos sólo por instantes (Dubet, 2013, pp. 170-181).

Lo descrito hasta el momento muestra que el concepto de vulnerabilidad social está construido a partir de la pérdida de centralidad del trabajo asalariado, que había sido el principal organizador de la vida social al hacer coincidir las posiciones con las subjetividades, las creencias y los significados acerca de una justicia basada en los derechos derivados de las posiciones laborales.

Por otro lado, en la sociedad del trabajo, la perspectiva de género estaba vinculada con la socialización o aprendizaje de determinados roles para los hombres y las mujeres a través de instituciones como la familia y la escuela, contexto en que la desigualdad de género se explicaba a partir de una socialización diferenciada que supuestamente era necesaria para la construcción de una identidad masculina y una femenina (Giddens, 2010, p. 635).

Lo anterior cambió con la expansión de la vulnerabilidad social -provocada por la generalización del trabajo precario, temporal y de bajos ingresos- como una nueva vía para 
reproducir la desigualdad de género (Ehrenreich, 2014, pp. 211-212). En México, la participación económica femenina ha estado más relacionada con el autoempleo precario y el trabajo doméstico con baja remuneración, o con un empleo sin salario, considerado como una ayuda familiar. Esta situación ha aumentado la vulnerabilidad de las mujeres pobres (Ortiz, 2014, pp. 56-58), aunque el orden social heterosexual también reproduce la vulnerabilidad a las mujeres mediante la violencia. Por ejemplo, en México, entre 1985 y 2013, 44646 mujeres murieron víctimas de homicidio. ${ }^{1}$

\section{Precariedad social y declive institucional}

La vulnerabilidad social conduce a la precariedad social mediante la transferencia de los riesgos y la inseguridad a los individuos, que antes eran atendidos por el sistema de bienestar estatal. La precariedad social nombra una realidad que, según Standing (2013, pp. 17-19), se caracteriza por el subempleo y los bajos salarios, favorables a la expansión de la pobreza y la desigualdad social.

Por otro lado, el desempleo también sería una manifestación de la declinación de la institución de bienestar organizada a través del trabajo. El aumento del desempleo ha afectado los principios organizativos de las instituciones, como la familia y la escuela, que han ayudado a los individuos a la construcción de su identidad social (Gilbert Ceballos, 2012, pp. 177-192).

El precariado ${ }^{2}$ ha surgido en un contexto social fragmentado, donde la desigualdad social tiene diversas causas (Dubet, 2000, p. 14). Al precariado lo integran individuos que sufren el desempleo y que están más cerca de la desa-

I. Los datos provienen de la Encuesta nacional sobre la dinámica de las relaciones en los hogares, 2013 (Muñoz, 20l4, p. 19).

2. La palabra precariado fue usada en los años ochenta del siglo XX por los sociólogos franceses para nombrar a los trabajadores temporales o estacionales. 
filiación social, es decir, en un tránsito hacia su conversión en parias, situación en que ya no existen lazos sociales, sino abandono por parte de las instituciones (Polavieja, 2003). Por tanto, el precariado es un individuo y no un sujeto social, porque su integración social es débil o nula (Standing, 2013, pp. 27-29).

En consecuencia, la precariedad significaría desempleo o empleo inseguro y con bajos ingresos, sin acceso a servicios como la salud y la educación, pero también, desde un punto de vista subjetivo, significaría la ausencia de una identidad que genere certeza sobre el porvenir. La precariedad también estaría integrada por otro hecho social: una discordancia entre la posición y el nivel de educación formal, es decir, la existencia de personas con educación formal elevada que desempeñan empleos de bajos ingresos y de baja productividad.

Como se puede visualizar, el concepto de precariedad social se ha formado a partir de la idea de seguridad y certeza, generada por un empleo de larga duración, estable y protegido por los derechos sociales, apoyado en el sistema de bienestar estatal.

De lo anterior se deduce que el individuo debería de afrontar los riesgos y la incertidumbre laboral utilizando su propio potencial o la ayuda limitada que le podía ofrecer una institución de socialización primaria como la familia.

La identidad del precariado no se puede formar a partir de su pertenencia a una colectividad o comunidad que le permita contar con prácticas estables, códigos éticos y normas de reciprocidad y fraternidad (Standing, 2013, p. 34). Por tal motivo, las posiciones laborales y las subjetividades dejan de coincidir y no existe posibilidad de que surja la solidaridad, sino un sentimiento generalizado de frustración y resentimiento: no hay futuro para el precariado, porque su hoy no tiene consecuencias en la construcción de lazos 
vinculantes a largo plazo a través de un empleo que para él sea temporal.

Como se ha mencionado, el precariado no tiene identidad ocupacional: debido a la expansión de la vulnerabilidad social, el empleo ha dejado de ser la base principal para la configuración de la identidad común. Por tal motivo, la crisis de identidad laboral, basada en la igualdad de posiciones, sólo existió en el periodo de auge del Estado de bienestar (Beck, 2001, pp. 13-29).

Sin embargo, según Standing (2013, pp. 35-36), el precariado no es homogéneo, a pesar de contar sólo con empleos ocasionales, lo que ha afectado más a los jóvenes aunque algunos han encontrado un pasatiempo en los cibercafés, mientras esperan acceder a otro empleo temporal. La misma espera comparte la madre soltera joven que desarrolla una actividad remunerada e inestable y que está obligada a sobrevivir en la casa de sus familiares.

Desde otro punto de vista, el precariado sería un residente, pero no un ciudadano, pues no tiene derechos que creen sentido de pertenencia con la sociedad, a pesar de realizar alguna actividad económica con bajas remuneraciones. Es decir, su condición de precariado niega su ciudadanía, lo que Logiudice (2007, pp. 49-51) visualiza como un individuo que se incluye en el orden legal o jurídico sólo por su situación de exclusión, ${ }^{3}$ es decir, como simple vida biológica con diferentes carencias que pueden ser la causa de alguna intervención estatal para asistirlo o criminalizarlo. La criminalización estatal es otra vía para castigar a los individuos que han sido despojados de sus derechos ciudadanos y de sus atributos sociales al considerarlos como un peligro para el orden estatal, justificando el uso de la

3. La palabra exclusión no significa estar fuera de la sociedad, sino ruptura de lazos con la sociedad, lo que crea individuos abandonados, desprotegidos por las instituciones estatales. 
fuerza estatal para su castigo o para terminar con su vida (Matthews, 2003, pp. 21-50).

En suma, el precariado es resultado de un proceso que indica la forma en que los individuos se precarizaron en el momento que perdieron sus atributos sociales y sus derechos ciudadanos, lo que los privó de su capacidad y control para distinguir lo útil de lo inútil, en un proceso en el cual las normas institucionales no influyen en sus comportamientos ni evitan su frustración y ansiedad (Standing, 2013, pp. 40-51).

En el contexto descrito, al parecer las instituciones no han podido detener el proceso de precarización a pesar de que son organizaciones, costumbres, hábitos, reglas, en otras palabras, formas de ser y pensar, que abarcan a la vida social (Dubet, 2006, pp. 30-31), sobre todo, porque producen reglas y decisiones legítimas, aunque Searle (2012, pp. 44-45) distingue entre reglas constitutivas y reglas regulativas: las primeras intervienen en la formación de condiciones o posibilidades para la realización de ciertas actividades, y las segundas regulan actividades y conductas.

Entonces, el estudio de las instituciones se puede hacer a través de sus programas, que designan un tipo particular de socialización: "[...] una forma específica de trabajo sobre los otros [...] se realiza ante todo por una interiorización de lo social, por una interiorización de la cultura que instituye a los actores sociales como tales" (Dubet, 2006, p. 32). El programa institucional es, entonces, un proceso social que convierte los valores en acción y subjetividad para la ejecución de una actividad específica y profesional. Dicha socialización forma una vocación que da paso a un individuo socializado y a un sujeto autónomo.

Por otro lado, se debe de considerar que algunas crisis de las instituciones han sido provocadas porque los programas de estas han perdido su capacidad de integración social, y porque con el auge del modelo económico neoliberal se ha 
buscado sustituirlos a través del establecimiento de una integración sistémica, realizada por el mercado y favorable al liberalismo económico (Dubet, 2006, pp. 70-71). La crisis de los programas institucionales de integración social tiene que ver con el auge de las actividades laborales informales y con la multiplicación de las situaciones de vulnerabilidad social y de precariedad, que han impulsado el surgimiento de sociedades fragmentadas.

Las sociedades fragmentadas se reproducen por la existencia de diversas actividades económicas de subsistencia e informales realizadas por los vulnerables y precarios (Mingione, 1993, pp. 113-137). En consecuencia, ante el avance de la vulnerabilidad y la precariedad, la crisis de las instituciones (o de sus programas de socialización) ha afectado a los jóvenes, que no encuentran relación entre las instituciones y sus condiciones de vida ni sentido a lo que viven y sufren, lo que significa que existe una debilidad de parte de las instituciones para dotar de atributos sociales a una diversidad de vidas juveniles.

La vulnerabilidad de la juventud mexicana se puede observar desde el aspecto laboral y escolar: seis de cada diez jóvenes trabajaban en el sector informal, y sus salarios son bajos porque el $33.1 \%$ gana de dos a tres salarios mínimos al día; el promedio de escolaridad es el nivel secundaria, y sólo el 18\% de los jóvenes tiene educación superior. Esto significa que existe una desigualdad de ingresos y de educación que reproduce la desigualdad de género, porque el $72.4 \%$ de los jóvenes que pertenecen al decil de los hogares de ingreso más alto asisten a la escuela, pero solamente asiste a la escuela el 40\% de los jóvenes del decil de hogares con ingreso más bajo (Carbajal, 2014, p. 28).

Por su parte, el desempleo, considerado como una expresión de la precariedad, afecta más a los jóvenes: la tasa de 
desocupación juvenil es de $8.4 \%$, es decir, el doble de la tasa de desocupación total. ${ }^{4}$

La vulnerabilidad también afecta a los jóvenes mexicanos que cursan estudios universitarios, ya que su tasa de desocupación es más alta (4.6\%) en comparación con la de los jóvenes que sólo poseen formación básica (3.5\%). Por otro lado, la precariedad tiene su manifestación entre la población juvenil que no estudia ni trabaja, cerca del $22 \%$, lo que visto desde el punto de vista de género significa que por cada hombre joven que no estudia ni trabaja existen tres mujeres que se encuentran en la misma situación, aunque realizan tareas domésticas o de cuidado de sus hijos. ${ }^{5}$

\section{La vulnerabilidad juvenil en la Ciudad de México}

El concepto de género tiene validez para el análisis porque considera que existe una construcción social, con sus significados particulares, sobre la distinción entre lo masculino y lo femenino, lo que reproduce una desigualdad de acuerdo con los atributos sociales que se les confieren al hombre y a la mujer (Urteaga, 2010, p. 42).

Por tal motivo, la construcción social del género nos conduce a considerar la manera en que surgen las identidades juveniles articuladas respecto de la sexualidad, y a su vez nos ayuđa a comp̄render tos problēmas quē las instituciones no resuelven y que afectan a los jóvenes, como el embarazo y maternidad adolescente, la salud reproductiva y el aborto.

El concepto de género también nos permite explicar la construcción cultural del cuerpo a través del grupo o del colectivo mediante rituales, estilos de vida, consumo de

4. Los datos provienen del reporte Calidad de vida de los jóvenes en México elaborado por el Instituto Nacional de Estadística y Geografía (INEGI), en 2013 (Carbajal, 20I4, p. 28).

5. Los datos son del documento Panorama educativo 2014, editado por la Organización para la Cooperación y el Desarrollo Económico (OCDE) (Poy, 20I4, p. 40). 
algunos tipos música, la adopción de ídolos y la reafirmación de los roles machistas en las reuniones grupales o en líderes de algunos grupos juveniles (Urteaga, 2010, p. 43).

En el diseño de la masculinidad y de la feminidad intervienen elementos del mercado, pero también, desde un punto de vista general, elementos propios que comparten los miembros del grupo juvenil para distinguirse de los demás, mostrando que las instituciones como la familia y la escuela han perdido no sólo su atractivo, sino su influencia en la configuración de los comportamientos juveniles (Olvera, 2014, pp. 56-58).

Los comportamientos juveniles de los estratos socioeconómicos pobres que habitaban la periferia de la Ciudad de México en la década de los años ochenta del siglo pasado fueron analizados como eventos públicos relacionados con la criminalidad, debido a que dejaron de reproducirse solamente en espacios privados de la vida social (García Robles, 2013). En dicho contexto, se construía un colectivo que generaba reglas y requisitos de ingreso, llamado banda, como medio de protección a un entorno cuya violencia tenía diversas causas y se reproducía en la familia, la escuela y hasta en las autoridades, que criminalizaban a dichos jóvenes para legitimar el uso de la fuerza policiaca (García Robles, 2013, pp. 8-20).

En el barrio, lo interesante de la banda fue la creación de una identidad cultural particular, la cual se pudo visualizar a través de la vestimenta y el consumo de estos jóvenes, sus actividades, comportamientos, expresiones verbales y manifestaciones escritas, esto último a su vez a través de la pinta de bardas (Valenzuela, 1988). Desde el punto de vista de Valenzuela (2002, p. 20), los grupos juveniles se caracterizan por la participación de sus integrantes en la conformación de poderes y liderazgos, códigos compartidos y una vida cotidiana similar, aunque su identidad ha sido utilizada para distinguirse de los otros grupos. 
En suma, el estudio de la identidad cultural juvenil ha permitido entender la solidaridad grupal-que las políticas públicas no creaban, lo que muestra su baja capacidad de integración social-. Esta consideración, al mismo tiempo, ayudó a comprender por qué los jóvenes no se identificaban con las instituciones sociales ni esperaban que intervinieran en el mejoramiento de sus condiciones de vida.

Desde el punto de vista del género, a través del mismo estudio se pudieron establecer las diferencias socioculturales entre los sexos, su uso biológico por parte de la política y su interpretación cultural a través de la aceptación de la desigualdad, sin embargo, la aceptación cultural de la diferencia no significa reafirmar la superioridad masculina ni su justificación por medio de la desigualdad social, que afecta tanto a los como a las jóvenes (Reguillo, 2002, pp. 160-162).

En consecuencia, el contexto en que vive la juventud mexicana no sólo está caracterizado por la desigualdad social, sino también por la débil integración social desarrollada a través de la sociedad, en la que surgen organizaciones particulares juveniles que logran articular sus vivencias con el grupo al que pertenecen, expresan su sentido o significado acerca de la vida social y viven, a su vez, la diferencia a través de la cultura que abarca los sexos.

Por otro lado, las percepciones de los jóvenes de la capital del país nos pueden ayudar a explicar el declive de instituciones (familia y escuela) en cuanto a su capacidad de integración social, la no identificación de los jóvenes con las mismas, y que la diferencia de sexos sigue siendo interpretada a través de los prejuicios machistas. ${ }^{6}$

6. El Gobierno de la Ciudad de México, en 2013, levantó 304000 encuestas en las dieciséis delegaciones en las que se divide su territorio. Sólo se recogió la opinión de las personas de ambos sexos que tenían entre catorce y veintinueve años, lo que significa que prevaleció el criterio de la edad para definir a los jóvenes, decisión criticada por los diferentes estudios sobre la juventud mexicana. 
La precariedad de los jóvenes de la capital se puede observar por dos vías: por el nivel de ingresos y por su limitado acceso a los servicios de salud públicos. En este caso, existe un fuerte vínculo entre ingresos bajos y débil protección estatal hacia los jóvenes ante las consecuencias negativas derivadas de la dinámica de la vida social, manifestadas en enfermedades o accidentes.

Por ello, el $45.1 \%$ de los jóvenes encuestados expresó que contaba para sus gastos solamente con MXN 1000.00 mensuales, mientras que sólo el 3.83\% dijo tener más de MXN 10000.00 al mes (Instituto de la Juventud del Distrito Federal, 2013, p. 25), lo que en realidad reflejaría una polarización social por ingresos. En otras palabras, sería uno de los factores que estarían contribuyendo a la reproducción de la desigualdad social mexicana.

Sus ingresos provienen básicamente de dos fuentes: de sus padres (50.49\% de los jóvenes) y de las becas que les otorga el Gobierno local a los jóvenes que estudian (14.08\% de los jóvenes). También, aunque en menor medida, se agrega a esto la ayuda otorgada por alguna institución privada (3.41\% de los jóvenes). Por otra parte, el $24.54 \%$ de los jóvenes señaló que sus ingresos eran generados por un empleo, el $3.26 \%$ por la pareja y el $2.4 \%$ por los amigos (Instituto de la Juventud del Distrito Federal, 2013, pp. 26, 28-29).

De esta manera, se puede observar que es menor la presencia institucional del Gobierno de la Ciudad de México en la generación de los ingresos de los jóvenes capitalinos, lo que puede interpretarse como una contribución a la generación de la vulnerabilidad y precariedad juvenil, pues el bienestar de los jóvenes es sostenido principalmente por los ingresos familiares y no por un empleo formal que les permita alcanzar su autonomía a través de las protecciones sociales gubernamentales.

\section{0}


Por otro lado, se puede también inferir de las cifras anteriores que los jóvenes de la capital enfrentan su precariedad a través de la familia, que sufre las consecuencias de la vulnerabilidad social, creada por la fragilidad de los derechos sociales. Otra manera de observar esto es mediante las cifras de acceso a los servicios de sanidad.

Por ejemplo, cuando los encuestados sufren alguna enfermedad recurren mayoritariamente a la automedicación (26.7\% de los encuestados) y como segunda opción van al servicio médico privado que se ofrece en algunas farmacias (17.66\%). Si sumamos ambos porcentajes, se tiene que el $44.36 \%$ de los encuestados no tiene protección pública médica, en tanto sólo el $17.85 \%$ de los encuestados es atendido por alguna institución médica pública como el servicio de salud del Gobierno de la Ciudad de México, el Instituto Mexicano del Seguro Social o el Instituto de Seguridad y Servicios Sociales de los Trabajadores del Estado (Instituto de la Juventud del Distrito Federal, 2013, pp. 80-81). Estas cifras indican una reproducción de la desigualdad social que no ha podido detenerse tampoco mediante las políticas públicas de sanidad.

Por lo anterior, no debe resultar extraño que la desconfianza que tiene la juventud de la Ciudad de México hacia las instituciones se exprese a través de un nulo interés por la política. En este caso, el 53.35\% de los jóvenes encuestados dijo que la política no le interesaba; sólo el 12.58\% dijo que se identificaba con una posición política de centro y el $23.3 \%$ se identificó con una posición de izquierda, tanto radical como moderada (Instituto de la Juventud del Distrito Federal, 2013, pp. 74-75). De esta manera, se puede afirmar que las instituciones políticas (partidos) no cumplen con la tarea de integrar a los jóvenes a la esfera de sus debates y mucho menos a sus espacios de representación, en consecuencia, pues, se puede decir que su representación tiende a ser más elitista. 
Los datos sobre un acceso limitado a los servicios de salud públicos y la desconfianza de los jóvenes hacia una representación política partidista muestran la baja capacidad de integración juvenil institucional que tiene el Gobierno de la capital, lo que se ha transformado en otra causa de la vulnerabilidad y precariedad, dos condiciones que influyen en la configuración de situaciones de desigualdad social.

Por otro lado, los apoyos sociales del Gobierno local han creado beneficiarios, pero no ciudadanos que puedan tener autonomía gracias a un soporte institucional. No resulta extraño que más de la mitad de los jóvenes encuestados (55.43\%) haya dicho que ha sido atendida alguna de sus carencias a través de algún programa gubernamental, pero exista un número importante de no beneficiarios (44.57\%). Esto último es resultado de la pérdida de universalidad de los programas de ayuda o asistencia social del Gobierno, que están enfocados a atender sólo algún tipo de carencias de algunos individuos definidos como beneficiarios (Instituto de la Juventud del Distrito Federal, 2013, p. 76).

Por otro lado, a pesar de la labor asistencialista del Gobierno de la ciudad y federal, los jóvenes conservan su percepción negativa sobre la atención que ofrecen las instituciones de Gobierno: sólo el 40.18\% de los encuestados dijo que la atención recibida en estas había sido regular, mientras que el $21.72 \%$ expresó que fue mala (Instituto de la Juventud del Distrito Federal, 2013, p. 79). En este caso la expresión regular haría alusión a la existencia de una atención institucional precaria, lo que también se relacionaría con una expansión de la vulnerabilidad, y, en consecuencia, con la reproducción de la desigualdad social juvenil.

Por otro lado, la encuesta de la que se extraen estos resultados fue aplicada siguiendo el criterio de género basado sólo en la distinción por sexo (de los encuestados, 51.14\% eran mujeres y $48.86 \%$ fueron hombres). A esta distinción se le agregó solamente la de la orientación sexual, que reflejó 
una inclinación mayoritaria por la heterosexual (hombre/ mujer). En este caso, el 80\% de los encuestados se declaró heterosexual, el 7\% homosexual y el $5 \%$ bisexual (Instituto de la Juventud del Distrito Federal, 2013, pp. 14, 21). Estos datos sólo indican que la visión juvenil dominante sobre su propia sexualidad es la relación heterosexual, negando las otras opciones a pesar de que las dos últimas se han ido generalizando a partir de la aprobación de la ley de matrimonios del mismo sexo en la capital del país.

En consecuencia, la distinción binaria de género (hombre/ mujer), vista desde la perspectiva de la desigualdad social, se ha abordado más como una discriminación social por la orientación sexual (hombre/hombre, mujer/mujer), o también derivada de la condición de ser mujer, porque las mujeres realizan un trabajo con bajo salario o sin remuneración, como es el caso de las labores domésticas o de ayuda, por ejemplo, en algún tipo de negocio familiar.

También en el tema de la primera relación sexual de los jóvenes capitalinos, sus respuestas mostraron su condición de vulnerabilidad y precariedad porque algunos tras dicha relación sexual se han convertido en padres o en madres, en un contexto donde todavía no tienen un empleo remunerado y tampoco han terminado el ciclo de estudios intermedios (secundaria/preparatoria). ${ }^{7}$

Por ello, se ha intentado relacionar la respuesta de la primera relación sexual de los jóvenes capitalinos con el aumento de los embarazos juveniles, aunque, en esta encuesta no quedó claro el vínculo. Sin embargo, el 28.17\%

7. En México, entre 2014 y 2016 , el embarazo adolescente aumentó un $8 \%$. Por ejemplo, en 2016, se registraron II 219 partos de mujeres de entre nueve y catorce años. El I7\% de esos partos ocurrió en un pueblo indígena. Dado esto, México tiene la tasa más alta de fecundidad de adolescentes entre los países miembros de la OCDE: setenta y seis nacimientos por cada I 000 mujeres de entre quince y diecinueve años de edad. Esto evidencia el fracaso de la Estrategia Nacional de Prevención del Embarazo en Adolescentes (Enapea), programa puesto en marcha por el presidente Enrique Peña Nieto en 2015 (Suárez, 2017, p. 9). 
de los encuestados señaló haber tenido su primera relación sexual entre los doce y diecisiete años, y el $25.31 \%$ entre los dieciocho y veinte años (Instituto de la Juventud del Distrito Federal, 2013, pp. 82-83).

Pero con base en otra información, una de las consecuencias del inicio de la vida sexual juvenil entre los doce y veinte años ha sido el aumento de los embarazos entre las mujeres adolescentes. Este acontecimiento se ha visualizado a través del análisis del crecimiento de la tasa de fecundidad de las adolescentes (Ramírez, 2014, p. 29).

La disminución de las oportunidades de educación y empleo para los jóvenes mexicanos ha sido otro factor que ha provocado que su único proyecto de vida sea el matrimonio, sobre todo para las mujeres. Sin embargo, también hay que considerar que un joven recién egresado de la educación superior universitaria gana en promedio MXN 8000.00 mensuales, lo que resulta insuficiente para costear una vida en pareja. Esto explicaría, entonces, el aumento de jóvenes con educación superior en el subempleo o desempleo, hecho que ha creado una mayor desigualdad en el país y también entre los jóvenes de la Ciudad de México (Guazo, 2014, p. A12). El empleo, entonces, se ha debilitado como un elemento de integración social: solamente el 32.48\% de los encuestados dijo trabajar (Instituto de la Juventud del Distrito Federal, 2013, p. 37).

Como se señalaba anteriormente, la vulnerabilidad es sobrellevada por los jóvenes a través de ayuda de la familia y, en menor medida, de amigos. Esta es la causa de que los jóvenes sigan viviendo en la casa de sus padres: el $74.27 \%$ aún vive con los mismos y $10.47 \%$ lo hace con otros familiares, mientras sólo $7.38 \%$ vive con su pareja (Instituto de la Juventud del Distrito Federal, 2013, p. 97).

Dicho esto, no resulta sorprendente que la madre es la persona depositaria de mayor confianza juvenil: $36.42 \%$ de los encuestados dijo que su persona de mayor confianza era 
su madre, seguida por el padre (15.42\%) y los hermanos (12.63\%), mientras que en los profesores sólo dijo confiar el $2.06 \%$ de los encuestados (Instituto de la Juventud del Distrito Federal, 2013, pp. 68-69).

En el mismo tenor, existe otro hecho que ha provocado que la madre sea la persona en la que más confían los jóvenes: el aumento de las mujeres jefas de hogar a nivel nacional, con pareja o sin ella, resultado del aumento en la tasa de participación económica femenina - del 17\% en 1970, pasó a 44\% en 2012- (López Barajas, 2014, pp. 16-17).

Dicho aumento de la tasa de participación económica de las mujeres se puede interpretar como otro factor que incide en la reproducción de la desigualdad social, porque muestra que las mujeres, al convertirse en jefas de hogar o en madres solteras, no sólo son beneficiarias de algunos programas de asistencia social, sino que además realizan actividades laborales, aunque con bajos salarios, lo que se liga con la vulnerabilidad (débil integración institucional) y la precariedad (empleos temporales), situaciones en que la condición de mujer (género) evidencia su desventaja en comparación con la condición de ser hombre. ${ }^{8}$

Por otro lado, en el tema educativo, el nivel de estudios máximo alcanzado por los encuestados fue de secundaria y bachillerato (36.73\% y $34.09 \%$, respectivamente). Solamente el 7.43\% dijo poseer un grado de licenciatura (Instituto de la Juventud del Distrito Federal, 2013, p. 40). De esto se puede derivar que un número importante de los jóvenes encuestados, que solamente alcanzó estudios de nivel secundaria o preparatoria, sólo podría tener acceso a empleos precarios (donde predominan las bajas remuneraciones), no favorables para lograr metas de bienestar a través del consumo.

8. Las desigualdades de género relacionadas con el desarrollo de las profesiones están sustentadas en estereotipos bajo los cuales, por ejemplo, existen más mujeres enfermeras y trabajadoras sociales, y más varones ingenieros, por lo que, en consecuencia, los mejores pagados resultan ser estos últimos (Cruz, 20I4, p. 5I). 
Para entender las insuficiencias que presenta la educación, no debe limitarse la reflexión a un problema de cobertura, que existe en las universidades públicas, sino que se debe también pensar que la educación ha dejado de ser un medio de ascenso social, cuando antes permitía tener un empleo estable y con ingresos aceptables para ejercer un individualismo posesivo a través de la adquisición de, por ejemplo, una vivienda propia y un automóvil.

Los datos revisados señalan que las aspiraciones que emergen de una situación de empleo estable y con protecciones sociales se conservan entre los jóvenes encuestados, pero sin un respaldo concreto y firme: ${ }^{9}$ el $80.48 \%$ seguía pensando que estudiar era la mejor garantía para elevar su calidad de vida, mientras el $19.52 \%$ no estaba de acuerdo con dicha aspiración; el $26.61 \%$ aspiraba a cursar estudios universitarios y el $23.11 \%$ quería llegar hasta un grado de técnico superior universitario (Instituto de la Juventud del Distrito Federal, 2013, pp. 44-47).

Las aspiraciones juveniles originadas por una situación ideal de empleo estable y con goce de derechos sociales se expresaron como consumo vinculado a una calidad de vida socialmente aceptada: el $87.82 \%$ de los jóvenes aspiraba a tener una vivienda propia, aunque el $61.52 \%$ pensaba que no era fácil obtenerla (Instituto de la Juventud del Distrito Federal, 2013, pp. 99-101). Igualmente, señalaban verse como profesionistas en diez años (29.35\%), o casados y con hijos (20.14\%) (Instituto de la Juventud del Distrito Federal, 2013, p. 67).

Los deseos y las aspiraciones juveniles son otra evidencia, insistimos, de que no existe una correspondencia entre las mismas y las posiciones laborales o escolares debido a que existe un proceso o programa de socialización deficiente,

9. Esto ha creado una desigualdad social, donde existe una diferencia entre el empleo barato, los empleos fijos o de larga duración y los empleos temporales (Alcaide, 20I4, p. 8).

\section{6}


aunque la familia sigue siendo el refugio de la vulnerabilidad y la precariedad provocada por la consolidación del modelo económico neoliberal en México (Ros Bosch, 2013, pp. 15-23).

Por otro lado, aunque se puede afirmar que los jóvenes de la Ciudad de México tienen acceso a internet (80.05\% dijo tenerlo, contra $19.95 \%$ que señaló que no lo tiene) y lo consideran de vital importancia para su existencia (43.57\% lo señaló como indispensable y 29.40\% como básico) (Instituto de la Juventud del Distrito Federal, 2013, pp. 49-51), esto no anula la brecha digital, otra manifestación de la desigualdad social originada por el uso de la web más para socializar que para adquirir conocimientos y habilidades necesarias durante el proceso educativo (Benach y Pueyo, 2014).

En consecuencia, la trayectoria de la juventud que habita la Ciudad de México no conduce hacia la construcción de individuos autónomos, apoyados por las instituciones, lo que ha creado una situación de desvinculación entre el sentido o significado de sus acciones, creencias y aspiraciones, y las instituciones, que no los han dotado de atributos sociales. En otras palabras, las instituciones los dejan solos para que recurran a estrategias individuales de sobrevivencia, a los apoyos precarios de la familia, parientes o amigos, o la economía informal, lo que representa también una declinación de las propias instituciones (Pérez Islas, 2010, p. 83).

Por otro lado, la desigualdad entre hombres y mujeres, en el plano nacional, se puede también observar cuando la tasa de desempleo en el país ha sido más alta entre las segundas que entre los primeros: históricamente ha oscilado alrededor de $4.7 \%$ para los hombres y $6.8 \%$ para las mujeres (Vela, 2008, p. 47).

En el contexto descrito mediante todos estos datos, la violencia juvenil sería un proceso que también tiene su causa en la crisis o en el declive de las instituciones, que forman a la sociedad mexicana y muestran deficiencias para 
dotar a la mayoría de los jóvenes de una identidad social (Bourdieu, 1982, pp. 53-54).

En resumen, la vulnerabilidad social de la juventud de la Ciudad de México ha sido reproducida por la debilidad de los derechos sociales, gestionados desde el Gobierno local en la forma de programas de asistencia social que están basados en algunas carencias particulares de sus beneficiarios. También, la vulnerabilidad ha ido articulada con la condición del subempleo-desempleo (la precariedad), reproducida a su vez como una desigualdad social, sostenida en la identidad binaria masculina y femenina (género).

\section{Conclusiones}

El concepto de vulnerabilidad social ha permitido explicar la aparición de un sujeto precario con ingresos irregulares que no tiene un empleo de larga duración ni protecciones o derechos sociales. Sin embargo, el problema del precario no sólo puede ubicarse en el ámbito de los cambios o transformaciones que ha sufrido el trabajo, basado ahora en una igualdad de posiciones, sino también en las consecuencias negativas que se han ido presentando en las instituciones encargadas de generar integración social.

El agotamiento de los mecanismos de integración social se considera como una crisis o declive de las instituciones, pues estas no pueden dotar a los individuos de autonomía, es decir, de identidad social o atributos sociales para desarrollar sus potencialidades dentro de la sociedad. La no identificación de las subjetividades con las posiciones ha provocado que los individuos vivan con sufrimiento, angustia y ansiedad, y opten por vías informales de sobrevivencia o por la ejecución de actividades ilícitas.

Sin embargo, como se vio, si las subjetividades no coinciden con las condiciones objetivas de posibilidad, derivadas de las instituciones, ello no significa que los individuos 
renuncien a sus aspiraciones de mejorar sus condiciones de vida a través de instituciones como la escuela y el trabajo. Esta contradicción es resultado de la fuerza que conserva una creencia colectiva que no se diluye del imaginario social, ya que no hay alternativas reales que ofrezcan certeza para mejorar las condiciones de vida.

La vulnerabilidad-precariedad social es un proceso que emergió durante la crisis de la sociedad del trabajo, pero también se ha relacionado con la crisis de las instituciones, incapaces de apoyar el paso de la juventud a la adultez. Por otro lado, algunas de las percepciones u opiniones de los jóvenes de la ciudad capital revisadas se explicaron a través de dichos conceptos (vulnerabilidad y precariedad social), que mostraron su potencial para analizar la dislocación entre instituciones y realidades que viven dichos jóvenes y que algunos estudiosos han relacionado, por ejemplo, con un Estado débil, ausente, con baja capacidad para regular los comportamientos sociales.

Por otro lado, los datos revisados indican que, al parecer, la familia y la escuela, como espacios de reproducción de la desigualdad social, no han podido influir en un cambio del punto de vista de los jóvenes (hombres) respecto a la comprensión de que la noción de la diferencia funciona como una manera de reconocer a las mujeres su autonomía y sus derechos sobre el uso de su cuerpo y su reproducción. El concepto de género ayuda, en consecuencia, a entender la manera en que se organizan los grupos juveniles para usar sus atributos grupales, y podría dotar de derechos a sus integrantes, es decir, a las mujeres.

En consecuencia, la violencia que viven los jóvenes capitalinos responde a causas diversas, pero algunas de ellas son producto de la dislocación de las instituciones, que no pueden cumplir con su tarea de otorgar atributos sociales para soportar la autonomía de la juventud. 
Bibliografía

Agamben, G. (2007). Estado de excepción. Buenos Aires: Adriana Hidalgo.

Aguilar Gil, M. (20I0). La sociología: construcción categorial, objeto y método. Madrid:Tecnos.

Alcaide, C. (I6 de noviembre de 20I4). "Los cambios del mercado laboral”. El País. Negocios, p. 8.

Ariza, M., y De Oliveira, O. (200I). "Inequidades de género y clase. Algunas consideraciones analíticas”. Nueva Sociedad, 2(164), I- 15.

Bauman, Z. (2008). Archipiélago de excepciones. Comentarios de Giorgio Agamben y debate final. Buenos Aires: Katz Editores.

(2013). Vidas desperdiciadas. La modernidad y sus parias. Barcelona: Paidós.

Beck, U. (200I). "Políticas alternativas a la sociedad del trabajo", en U. Beck, et al. (auts.), Presente y futuro del Estado de bienestar: el debate europeo (pp. 13-29). Buenos Aires: Miño y Dávila, SIEMPRO, Secretaría de Tercera Edad y Acción Social, Ministerio de Desarrollo Social y Medio Ambiente.

Benach, E., y Pueyo, M. (20I4). ¿Una eternidad digital? Vida y muerte antes y después de internet. España: Milenio.

Bourdieu, P. (1982). Lecon sur la Lecon. París: Les Éditions De Minuit.

Butler, J. (20I4). El género en disputa. El feminismo y la subversión de la identidad. Barcelona: Paidós.

Carbajal, R. (I6 de enero de 20 I4)."Radiografía de la juventud". Reporte Índigo, p. 28.

Castel, R. (2004a). Las metamorfosis de la cuestión social. Una crónica del salariado. Buenos Aires: Paidós.

(2004b). La inseguridad social ¿Qué es estar protegido? Buenos Aires: Manantial.

(2010). Las transformaciones del trabajo, de la producción social y de los riesgos en un periodo de incertidumbre. 
Buenos Aires: Instituto Di Tella-Cátedra UNESCO-Siglo Bibliografía XXI editores.

y Haroche, C. (2003). Propiedad privada, propiedad social, propiedad de sí mismo. Rosario Santa Fe: HomoSapiens Ediciones.

Chul Han, B. (20I3). La sociedad de la transparencia. Barcelona: Herder.

Cruz, Á. (07 de noviembre de 20I4). "Persisten 'miles de formas' de desigualdad de género: UNAM. Mujeres responsables de la crianza de los hijos y las tareas domésticas". La Jornada, p. $5 \mathrm{I}$.

Donzelot, J. (2007). La invención de lo social. Ensayo sobre la declinación de las pasiones políticas. Buenos Aires: Nueva Visión.

Dubet, F. (2000). Les inégalités multipliées. París: Ediciones de I'Aube.

- (2006). El declive de la institución, profesiones, sujetos e individuos de la modernidad. Barcelona: Gedisa.

(20II). Repensar la justicia social contra el mito de la igualdad de oportunidades. Buenos Aires: Siglo XXI editores.

- (20I3). El trabajo de las sociedades. Buenos Aires: Amorrortu/editores.

Ehrenreich, B. (2014). Por cuatro duros. Cómo (no) apañárselas en Estados Unidos. Madrid: Capitán Swing Libros.

García Robles, J. (20I3). ¿Qué transa con las bandas? México: Editorial Porrúa.

Garland, D. (2007). Crimen y castigo en la modernidad tardía. Bogotá: Siglo del Hombre Editores-Universidad de los Andes-Pontificia Universidad Javeriana-Instituto Pensar. Giacomello, C. (20I3). Género, drogas y prisión. Experiencias de mujeres privadas de su libertad en México. México: Tirant Lo Blanch.

Giddens, A. (20I0). Sociología. Madrid:Alianza Editorial. 
Gilbert Ceballos,J.(20I2). Introducción a la sociología.Santiago de Chile: LOM Ediciones.

Guazo, D. (02 de noviembre de 20 I4).“Los jóvenes le huyen al matrimonio". El Universal, p. AI2.

Instituto de la Juventud del Distrito Federal (2013). Consulta de tendencias juveniles 20/3. Ciudad de México: Gobierno de la Ciudad de México.

Le Blanc, G. (2007). Vidas ordinarias. Vidas precarias. Sobre la exclusión social. Buenos Aires: Nueva Visión.

Logiudice, E. (2007). Agamben y el estado de excepción. Una mirada marxista. Buenos Aires: Herramienta.

López Barajas, M. (20।4). "Cargas de trabajo y corresponsabilidad social”. México Social, 3(46), I6-19.

Matthews, R. (2003). Pagando tiempo. Una introducción a la sociología del encarcelamiento. Barcelona: Ediciones Bellaterra.

Mingione, E. (1993). Las sociedades fragmentadas. Una sociología de la vida económica. Más allá del paradigma del mercado. Madrid: Ministerio de Trabajo y Seguridad Social.

Muñoz, A. (07 de septiembre de 2014). "Mueren siete mujeres al día víctimas de homicidio: datos de Inegi y la Ssa”. La Jornada, p. 19.

Ochando Claramunt, C. (2002). El Estado del bienestar. Barcelona:Ariel.

Olvera Romero, C. (20I4). Sobre el cuerpo. Ensayos sobre la estética contemporánea. México: Fontamara.

Ortiz, R. M. (20I4)."Sentido de agencia y empoderamiento de la mujer a través del proceso de consolidación habitacional en colonias populares de origen irregular. Guadalajara (México), 1970-2009”. Anuario de Investigación del Posgrado de Urbanismo, (0), 55-75.

Paugam, S. (2007). Las formas elementales de la pobreza. Madrid:Alianza Editorial.

Pérez Islas, J. A. (20I0)."Las transformaciones en las edades sociales. Escuela y mercados de trabajo”, en R. Regui- 
Ilo (coord.), Los jóvenes en México (pp. 52-89). México: Bibliografía

Consejo Nacional para la Cultura y las Artes-Fondo de Cultura Económica.

Polavieja, J. G. (2003). Estables y precarios. Desregulación laboral y estratificación social en España. Madrid: CIS-Siglo XXI Editores.

Poy, L. (20 de mayo de 20I4). "Favorece mercado laboral a gente con educación básica: OCDE”. La Jornada, p. 40. Ramírez, N. (20I4). "La prevención fallida”. México Social, 3(46), 29-3I.

Reguillo, R. (2002).“Cuerpos juveniles, políticas de identidad”, en C. Feixa, F. Molina, y C. Alsinet (eds.), Movimientos juveniles en América Latina. Pachucos, malandros, punketas (pp. I5I-165). Barcelona:Ariel.

(20I2). Culturas juveniles. Formas políticas de desencanto. Buenos Aires: Siglo XXI.

Ros Bosch, J. (20I3). Algunas tesis equivocadas sobre el estancamiento económico de México. México: El Colegio de México.

Rosanvallon, P. (20I2). La sociedad de iguales. Buenos Aires: Manantial.

Searle, J. R. (20I2). La construcción de la realidad social. Barcelona: Paidós.

Standing, G. (20|3). El precariado. Una nueva clase social. Barcelona: Pasado \& Presente.

Steger, M., y Roy, R. (20I I). Neoliberalismo. Una breve introducción. Madrid:Alianza Editorial.

Suárez, K. (26 de octubre de 2017). "El embarazo de adolescentes mexicanas crece un $8 \%$ en dos años hasta alcanzar más de 1000 nacimientos al día. Niñas que crían a otras niñas”. El País, p. 9.

Urteaga, M. (20I0).“Género, clase y etnia. Los modos de ser joven”, en R. Reguillo (coord.), Los jóvenes en México (pp. |5-5I). México: Consejo Nacional para la Cultura y las Artes-Fondo de Cultura Económica. 
Bibliografía
Valenzuela,J.M. ( 1988). ¡A la brava ése!, cholos, punks y chavos banda.Tijuana: El Colegio de la Frontera Norte.

(2002)."De los pachucos a los cholos. Movimientos juveniles en la frontera México-Estados Unidos”, en C. Feixa, F. Molina, y C.Alsinet (eds.), Movimientos juveniles en América Latina. Pachucos, malandros, punketas (pp. I I-34). Barcelona: Ariel.

(2009).El futuro ya fue. Socioantropología de I@s jóvenes en la modernidad. México: El Colegio de la Frontera Norte-Casa Juan Pablos.

Valero Matas, J. A. (2009). Una mirada a la sociología desde las ciencias sociales. Madrid:Tecnos.

Vela, F. (2008). "Cambios en la estructura por edad y su efecto en el desempleo de los jóvenes urbanos en México", en F. Vela (coord.), La dinámica demográfica y su impacto en el mercado laboral de los jóvenes (pp. 47-87). México: Universidad Autónoma Metropolitana Xochimilco.

Vendrell, J. (20I3). La violencia del género. Una aproximación desde la antropología. México: Juan Pablos Editor, Universidad Autónoma del Estado de Morelos.

Wacquant, L. (2000). Las cárceles de la miseria. Buenos Aires: Manantial.

Zaremberg, G. (20I3). El género en las políticas públicas. Redes, reglas y recursos. México: FLACSO México. 